\title{
Conservative treatment using a sponge cast for transfer fractures in nursing home patients
}

This article was published in the following Dove Press journal:

Clinical Interventions in Aging

\author{
Hong Man Cho (iD ${ }^{1}$ \\ Joon Soo $\mathrm{Ha}$ (D) $^{2}$ \\ Jae Woong Seo $\mathbb{D}^{1}$ \\ Hyun Ju Lee (iD ${ }^{\prime}$ \\ Sun Do Kim (D) \\ Hyochoon Lee $\mathbb{D}^{\prime}$ \\ Hyung Bae Park $\mathbb{D}^{3}$ \\ 'Department of Orthopaedic Surgery, \\ Gwangju Veterans Hospital, Gwangju, \\ Korea; ${ }^{2}$ Department of Orthopaedics, \\ King's College Hospital, London, UK; \\ ${ }^{3}$ Department of Biology, College of \\ Chonbuk National University, Jeonju, \\ Korea
}

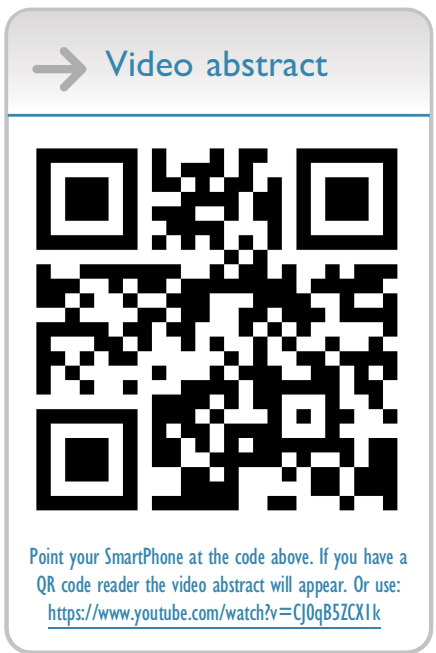

Correspondence: Hong Man Cho Department of Orthopedic Surgery, Gwangju Veterans Hospital, 99

Cheomdanwolbong-ro, Gwangsan-gu,

Gwangju 62284, Korea

Tel +82626026162

Fax +82626026936

Email chm1228@hanmail.net
Background: Transfer fractures in the lower limbs of bedridden and chair-bound nursing home patients can result from trauma induced by the usual lifting, moving, turning, or transferring maneuvers. Treatment entails immobilization for pain control and position change; however, splints/hard casts increase the risk of pressure sores. Therefore, we evaluated the use of a sponge cast.

Materials and methods: Between March 2011 and October 2017, 17 patients with a lower limb transfer fracture due to transferring maneuvers in a nursing home were recruited. We evaluated the improvement in pseudo-motion and divided the patients as having bony union, fibrous union, or remaining pseudo-motion. We also investigated the occurrence of pressure sores due to immobilization up until the final follow-up.

Results: Femur fractures occurred in 15 patients and lower leg fractures in two. Six of the 15 femur fractures were periprosthetic (four hip arthroplasty and two knee arthroplasty). Pseudo-motion was improved in 15 of 17 cases, within an average of 17.3 weeks for the improvement (14-23 weeks; bony union: 11 cases and fibrous union: four cases). Pseudomotion remained in two cases: one periprosthetic fracture around the knee arthroplasty and the other, a femur neck fracture. No pressure sores occurred.

Conclusions: A sponge cast appears to be one of the effective treatment options available for bedridden or chair-bound patients with a lower limb fracture due to its low risk of complications and satisfactory clinical results.

Keywords: transfer fracture, long bone fracture, conservative treatment, bedridden, immobile patients

\section{Introduction}

Transfer fractures of long bones in bedridden and chair-bound nursing home patients are often the result of trauma induced by the usual lifting, moving, turning, or transferring maneuvers. These maneuvers aim to prevent muscle retraction and decreased range of motion, as well as other complications such as bedsores and blood circulation problems, and are used to transfer patients from a chair or wheelchair to the bed or the toilet. ${ }^{1}$ Transfer fractures occurring in the lower extremities can be easily positioned by performing anatomical reduction and stable fixation through surgical treatment, which shortens the period of bed rest so that various complications associated with a long period of bed confinement can be prevented. ${ }^{2}$ However, if the patient is elderly or immobile or if the patient's physical status is poor, it is difficult to obtain good results after the surgery. Therefore, in such cases, the medical staff rarely recommends surgical treatment, which is also often preferred by the patients' guardians. ${ }^{3}$ In non-surgical cases, immobilization is required for pain control and 
positional change. However, it is difficult to manage the patient's hygiene when using a typical splint or a cast made of hard material, leading to a risk of pressure sores. In the present study, we sought to evaluate the results of transfer fractures in bedridden and chair-bound patients using a sponge cast.

\section{Materials and methods Study population}

Between March 2011 and October 2017, immobile patients in a nursing home or hospital who visited the Department of Orthopedic Surgery, Gwangju Veterans Hospital with a lower limb transfer fracture due to transferring maneuvers were assessed for recruitment to the study. In cases of patients who were deemed at high risk for surgery and unable to be maintained under anesthesia after collaborative consultation with the departments of internal medicine and anesthesiology, the guardian was informed of the outcome directly by the surgeon. The advantages and disadvantages of surgical treatment and conservative treatment were explained in detail, and the treatment method was decided after the family members had thoroughly discussed the options. Of the 18 patients who refused the surgical treatment and selected the conservative treatment, 17 patients, excluding one patient who died within 3 weeks of commencing the study, underwent treatment with the sponge cast. The clinical and radiological results were subsequently analyzed.

Informed consent was obtained verbally prior to the study. This was reviewed and approved by the Institutional Review Board (IRB) of Gwangju Veterans Hospital (IRB approval number: GJVH-IRB No. 2017-12-2) and the study was carried out in accordance with the latest version of the Declaration of Helsinki. Subjects of this study were first selected from ICD codes in the EMR system.

\section{Sponge cast procedure}

The sponge has a multi-pore structure; therefore, it is not heavy and allows ventilation. The material is also passively deformable but has the strength to resist gravitational forces (Figure 1A). The patient was placed in the supine position on a sponge with a width of $60 \mathrm{~cm}$ and thickness of $5 \mathrm{~cm}$. The length of the sponge ranged from the big toe to the iliac wing if the femur was fractured, from the big toe to the proximal thigh if the proximal tibia was fractured, and from the big toe to the tibial tuberosity if the distal tibia was fractured (Figure 1B). After the sponge was cut to enable the broken limb to be wrapped circularly (Figure 1C), the limb was wrapped with multiple bandages $(6 \times 12 \mathrm{~cm})$ (Figure 1D) and then fixed with an adhesive elastic bandage (Peha Haft 8x20, Hartmann, Berlin, Germany) by winding circularly (Figure 1E). In the case where the fixation of the sponge was necessary up to the iliac crest, it was annularly fixed to the pelvis in a state of continuity with the sponge placed in the back. A semicircular opening (diameter of $15 \mathrm{~cm}$ ) was made around the anus for hygiene management (Figure 2A), and sanitary management was implemented by applying adult pads outside the sponge (Figure 2B). If the patient had ankylosis in the joint and if it was necessary to retain the joint in a fixed position, part of the sponge cast was cut into a wedge shape (Figure 2C) and fixed to maintain the angle of ankylosis (Figure 2D). When it was judged that the range of joint movement should be limited, a hard splint was placed on the back or side of the sponge at an appropriate angle and length and was fixed with an elastic bandage (Figure 2E).

\section{Cast change and paramedical services}

For the prevention of sores and to maintain patient hygiene management, the authors trained the caregiver directly for the position change methods whenever the patient visited and a nursing team in the nursing home was required to turn the patient every $4 \mathrm{hrs}$. The status of patients visiting a nursing service was examine once a week. This was essential to prevent pressure sores from developing. In addition, the patient's toiletry needs were managed while the patient was in bed for at least the first 6 weeks of treatment. If the fixed lower limb became obviously loose in the sponge and if it was judged that the sponge was contaminated with a foreign substance that may adversely affect the patient, the sponge was replaced immediately at the hospital. In other cases, the sponge was replaced after the patient's condition was checked in accordance with the hospital's follow-up visiting schedule. The sponge casts were removed when there was clinically-confirmed absence of pseudo-motion and pain and in the case of union of the fracture site, which was radiologically confirmed. To confirm the recurrence of pseudo-motion when the union was not complete radiologically, follow-up was performed at 4 and 8 weeks after removal.

\section{Clinical and radiological measurements}

Sponge casts were applied every 2 weeks for the first 6 weeks and every 4 weeks thereafter. Clinical evaluation 


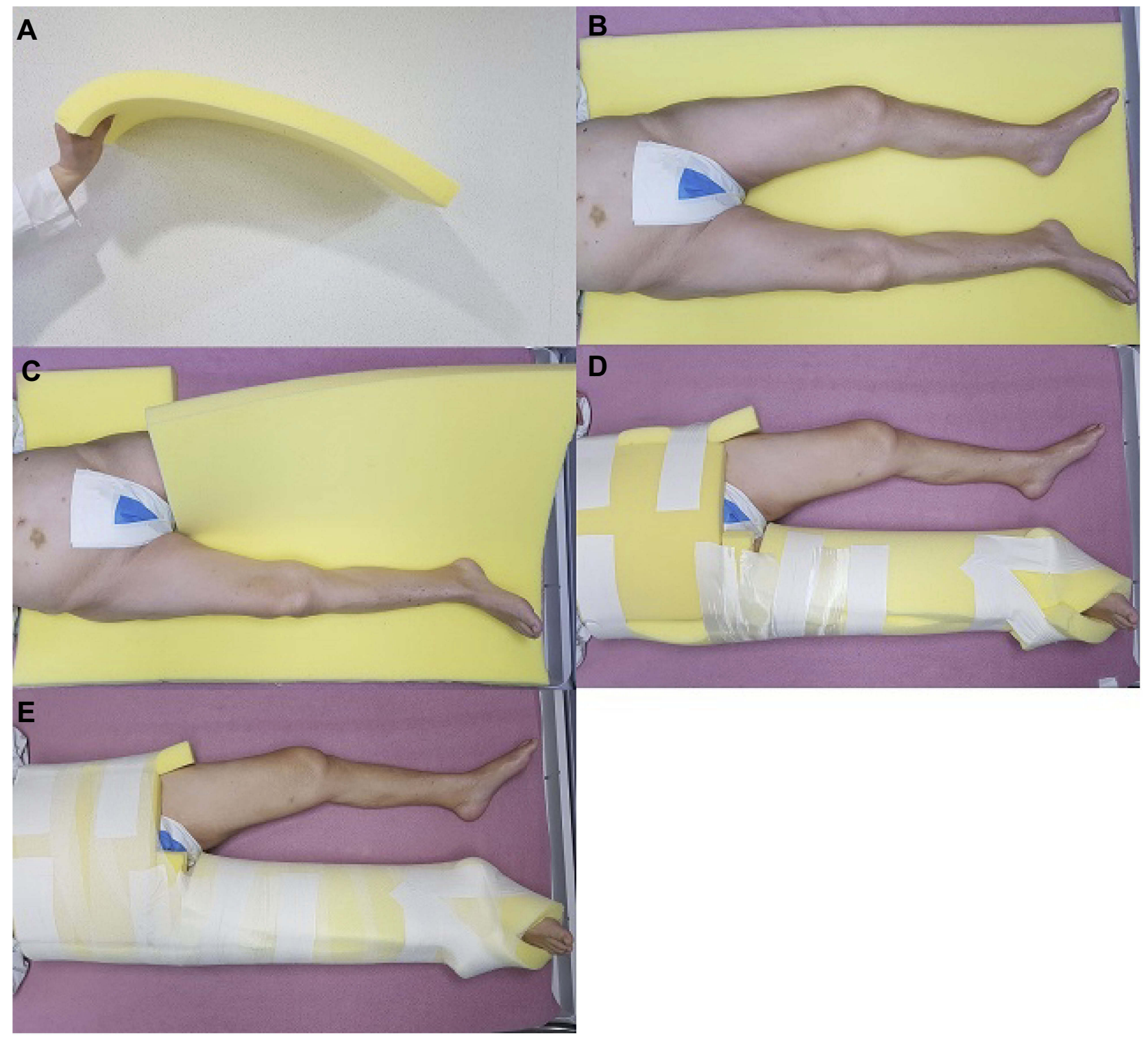

Figure I (A) The sponge has a multi-pore structure; thus, it is light, easily ventilated, and can be passively deformed but has the strength to resist gravitational forces. (B) The patient was placed in a supine position on the sponge. The length of the sponge ranged from the big toe to the iliac wing if the femur was fractured. (C) The sponge was cut to allow the broken limb to be wrapped circularly. (D) The sponge cast was fixed with multiple bandages. (E) The adhesive elastic bandage was wrapped circularly and fixed.

was performed to assess pressure sores and pseudo-motion. The presence of pressure sores was assessed in the whole body, and the size of the pressure sores that occurred in patients after applying the sponge cast, regardless of severity, was analyzed. Pseudo-motion in patients with zero-totrace on the manual muscle testing grade (11 out of 17 cases) was measured by passively elevating the lower limb, and in patients with poor-to-fair on the manual muscle testing grade (6 out of 17 cases), by actively elevating the lower limb, besides passive measurement. To assess the effects of fixing and prolonged bed rest due to fracture of the lower limb, the symptoms that were assumed to be newly developed (eg, pneumonia and thrombosis) were compared with the condition of pre-fracture patients. Radiological determination of fracture healing (bony union) was performed based on the following: absence of false motion or tenderness at the fracture site; observation of three or four cortical bridges on the anteroposterior and lateral radiographs; and progressive loss of the fracture line. We classified the outcomes as into bony union, fibrous union, and remaining pseudo-motion.

\section{Results}

Of the 17 patients included in the study, 16 were female and one was male. The reason for the patients being in an immobile state was unknown in seven cases and was 


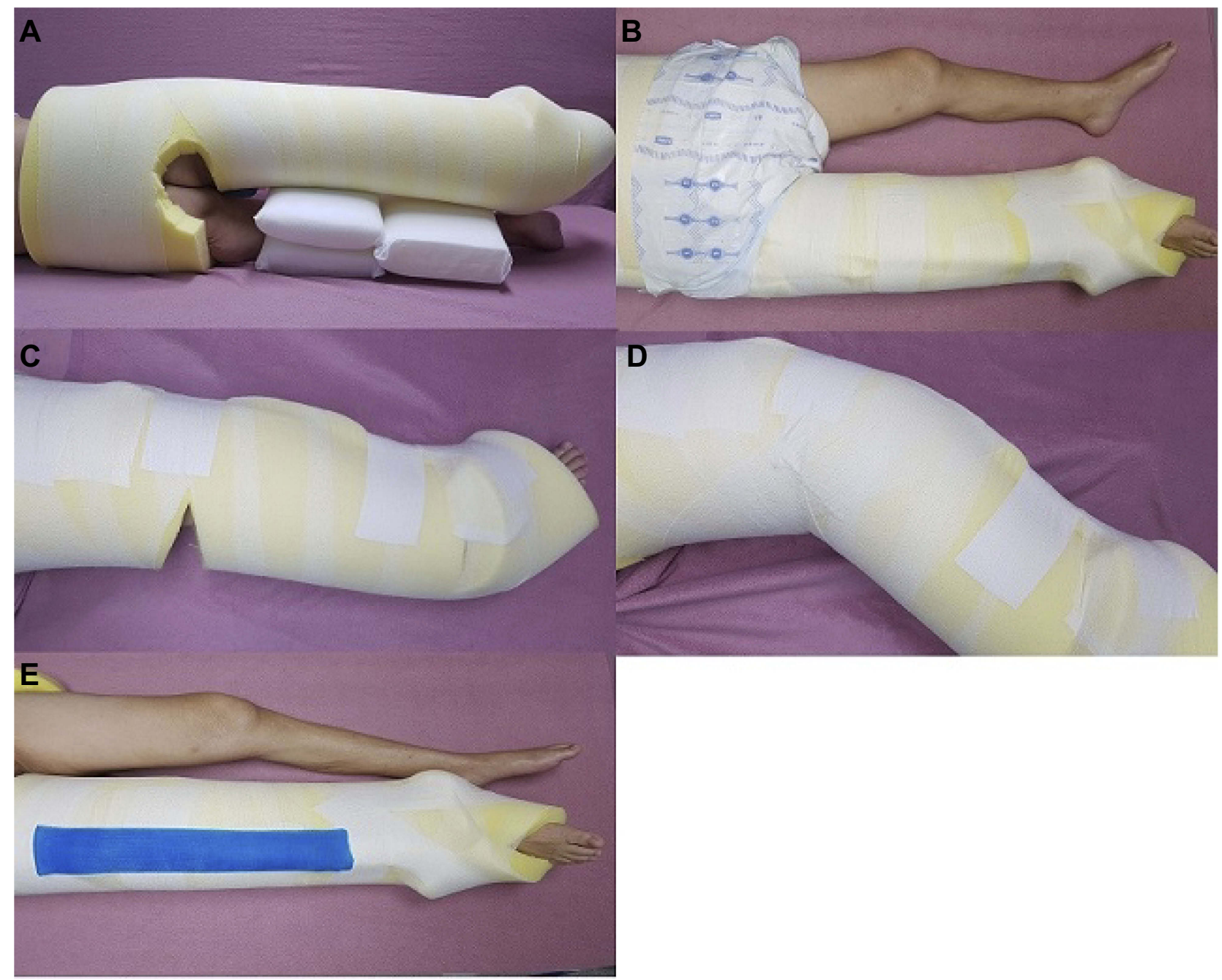

Figure 2 (A) When fixation of the sponge was necessary up to the iliac crest, it was annularly fixed to the pelvis in a state of continuity with the sponge placed in the back. (B) A semicircular opening was made around the anus for hygiene management. (C) Sanitary management was implemented by applying adult pads. (D) The front and back sides of each joint area were cut into wedge shapes to come into a functional position. (E) When it was judged that the range of joint movement should be limited, a firmly hardened splint was placed on the back or side of the sponge at an appropriate angle and length, which was fixed with an elastic bandage.

cerebrovascular disease in five cases. The mean age was 83.6 years (range, 38-90 years), and the mean body mass index was $19.9 \mathrm{~kg} / \mathrm{m}^{2}$ (range, $15.7-23.2 \mathrm{~kg} / \mathrm{m}^{2}$ ). The Singh index was evaluated preoperatively at the time of the procedure from the contralateral hip radiographs, and the average score was found to be 3.15 (range, 3-5). There were no patients who were able to use the wheelchair unaided before the fracture; nine patients could use a wheelchair with the help of others and eight were in a complete bedridden condition. Femur fractures occurred in 15 cases and lower leg fractures in two cases (Figure 3A-C). Six of the 15 femur fractures were periprosthetic (four were hip arthroplasty) (Figures $4 \mathrm{~A}-\mathrm{C}$ and $5 \mathrm{~A}-\mathrm{D}$ ), and two were around the knee arthroplasty. Of the two lower leg fractures, one was a mid-shaft fracture and the other, a distal tibia fracture. The mean follow-up period was 13.5 months, (range, 638 months) (Table 1).

\section{Clinical and radiological results}

Passively measured pseudo-motion was improved in 15 of the 17 cases within an average of 17.3 weeks (range, 14 23 weeks). Passive pseudo-motion remained in two cases; one periprosthetic fracture around the knee arthroplasty and one femur neck fracture. The patient with fracture around the articular joint above the knee joint was able to actively perform lower limb elevation before the injury. Passively, slight pseudo-motion was observed under the imaging amplifier; however, there was no pseudo-motion or pain in the active lower limb upon elevation, so the sponge cast was removed after 16 weeks of fixation and there was no progression of 


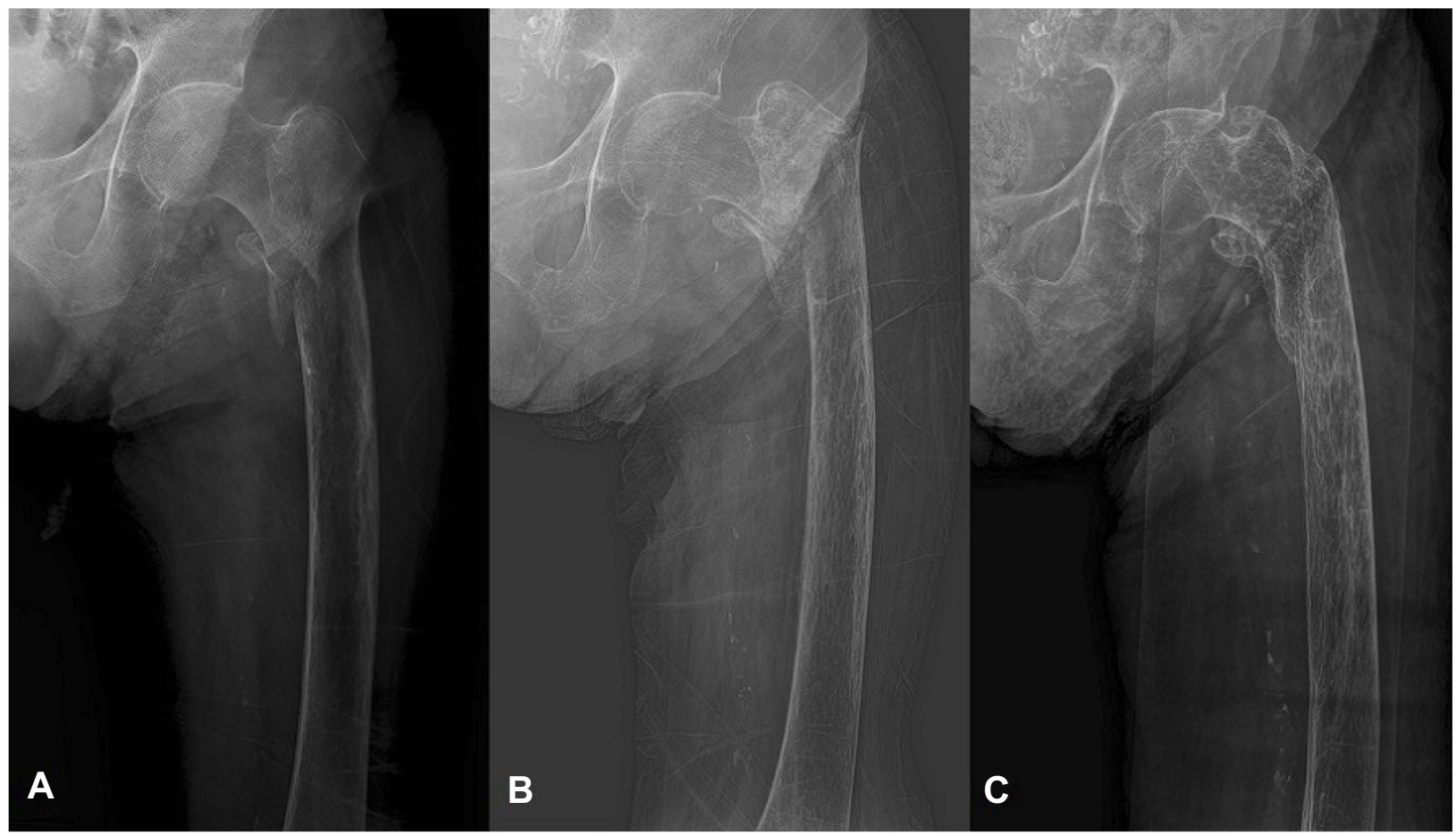

Figure 3 An 84-year-old woman was admitted to the hospital with a transfer fracture in the femur shaft. (A) On simple radiography, the right femur shaft shows a fracture with angulation and shortening. (B) A 10-week radiograph after the sponge cast was applied. (C) An I8-week radiograph after sponge casting, which shows union of the fracture.

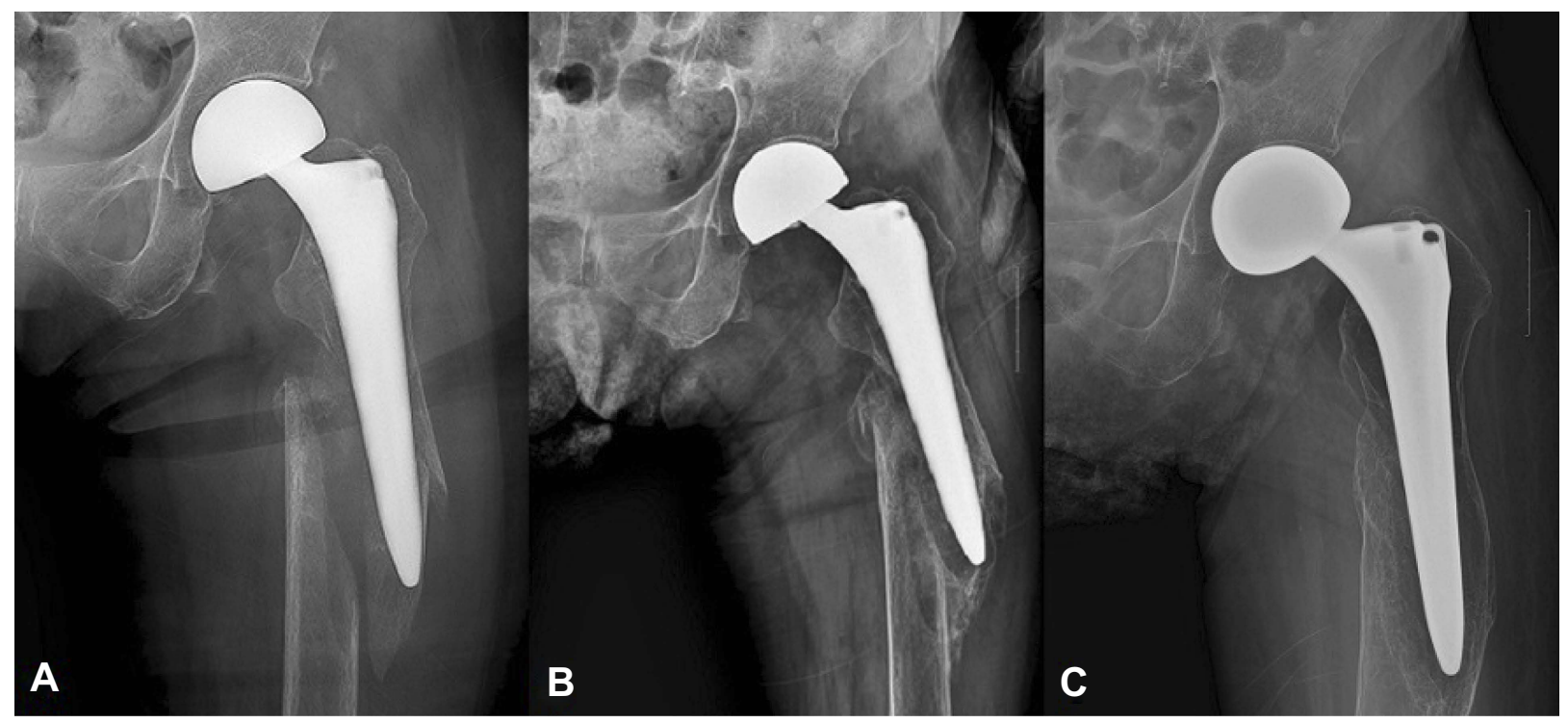

Figure 4 A 77-year-old woman was admitted to the hospital with a transfer fracture in the femur shaft. (A) On simple radiography, a fracture of the left femur around the femur stem with angulation and shortening was observed. (B) A 12-week radiograph after the sponge cast was applied. (C) A 20-week radiograph after sponge casting, which shows the union of the fracture.

pseudo-motion at follow-up (Figure 6A-C). The patients with a femoral neck fracture in which the pseudo-motion remained did not complain of pain during various movements such as position change or personal hygiene. There was no prominent pseudo-motion during the care process, and the cast was removed at 20 weeks. In all cases, no pressure sores occurred up until the final follow-up. Two patients developed aspiration pneumonia at 2 and 3 weeks after sponge casting, but both were treated with antibiotics and tube feeding for 10 days and improved. A radiologically complete bony union was 


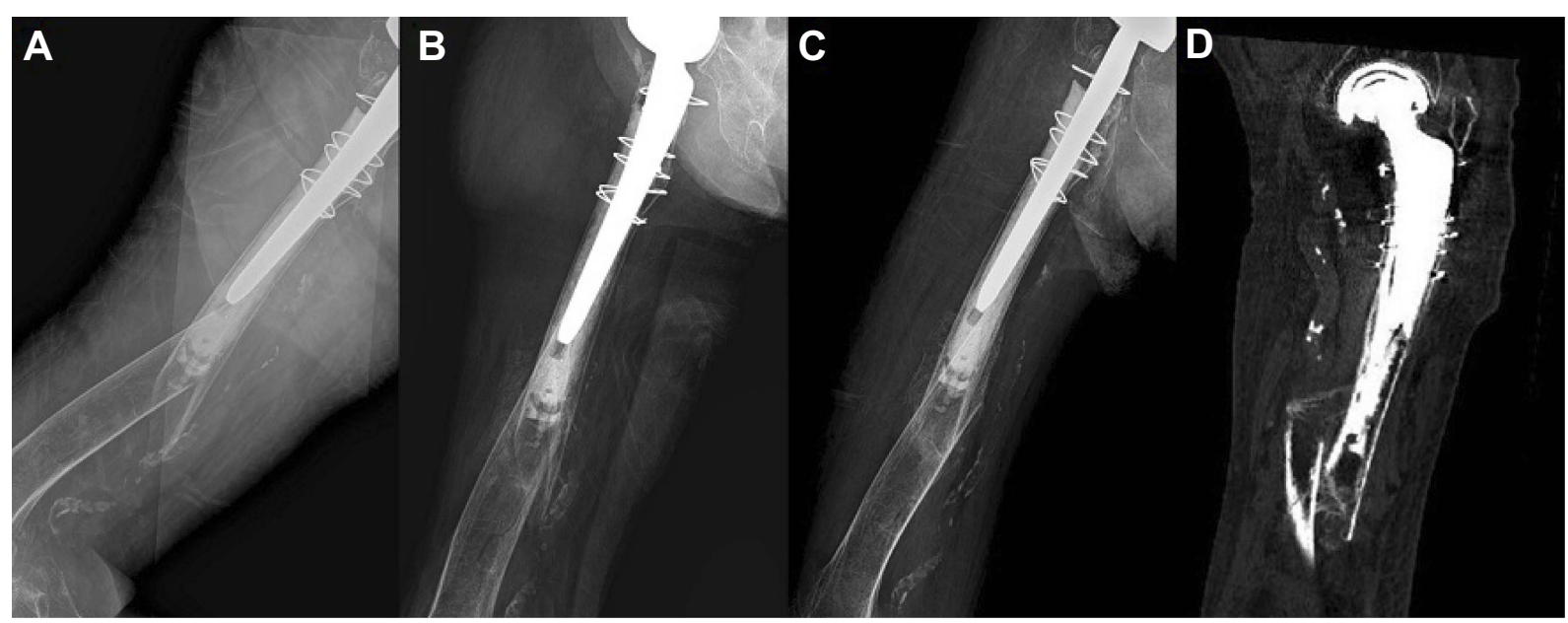

Figure 5 An 81 -year-old woman was admitted to the hospital with a transfer fracture in the femur shaft. (A) On simple radiography, a fracture of the left femur around the femur stem with angulation and shortening was observed. (B) A 12-week radiograph after the sponge cast was applied. (C) A 20-week radiograph after sponge casting, which shows the union of the fracture. (D) Computerized tomography of the femur.

Table I Demographic data

\begin{tabular}{|c|c|c|}
\hline \multicolumn{2}{|l|}{ Characteristic } & \multirow{2}{*}{$\begin{array}{l}\text { Number of cases }(n=17) \\
1: 16\end{array}$} \\
\hline Sex (male:female) & & \\
\hline \multirow[t]{4}{*}{ Reason for bedridden state } & Unknown (maybe aging process) & 7 \\
\hline & Cerebrovascular disease & 5 \\
\hline & Spinal cord injury & 3 \\
\hline & Brain injury & 2 \\
\hline Age (years) & & 86.3 (range: 38-90) \\
\hline Body mass index $\left(\mathrm{kg} / \mathrm{m}^{2}\right)$ & & 19.9 (range: 15.7-23.2) \\
\hline Singh index & & 3.15 (range: $3-5$ ) \\
\hline \multirow[t]{2}{*}{ Mobile status } & Completely bedridden & 8 \\
\hline & Dependent wheelchair ambulation & 9 \\
\hline \multirow[t]{7}{*}{ Fracture (fx) type } & Periprosthetic fx hip arthroplasty & 4 \\
\hline & Periprosthetic fx knee arthroplasty & 2 \\
\hline & Femur shaft $\mathrm{fx}$ & 5 \\
\hline & Femur intertrochanter $\mathrm{fx}$ & 2 \\
\hline & Femur neck fx & 2 \\
\hline & Lower leg mid-shaft $f x$ & 1 \\
\hline & Lower leg distal $\mathrm{fx}$ & 1 \\
\hline \multirow[t]{2}{*}{ Manual muscle testing } & Zero trace & 11 \\
\hline & poor $\sim$ fair & 6 \\
\hline Follow-up (months) & & I3.5 (range: 6-38) \\
\hline
\end{tabular}




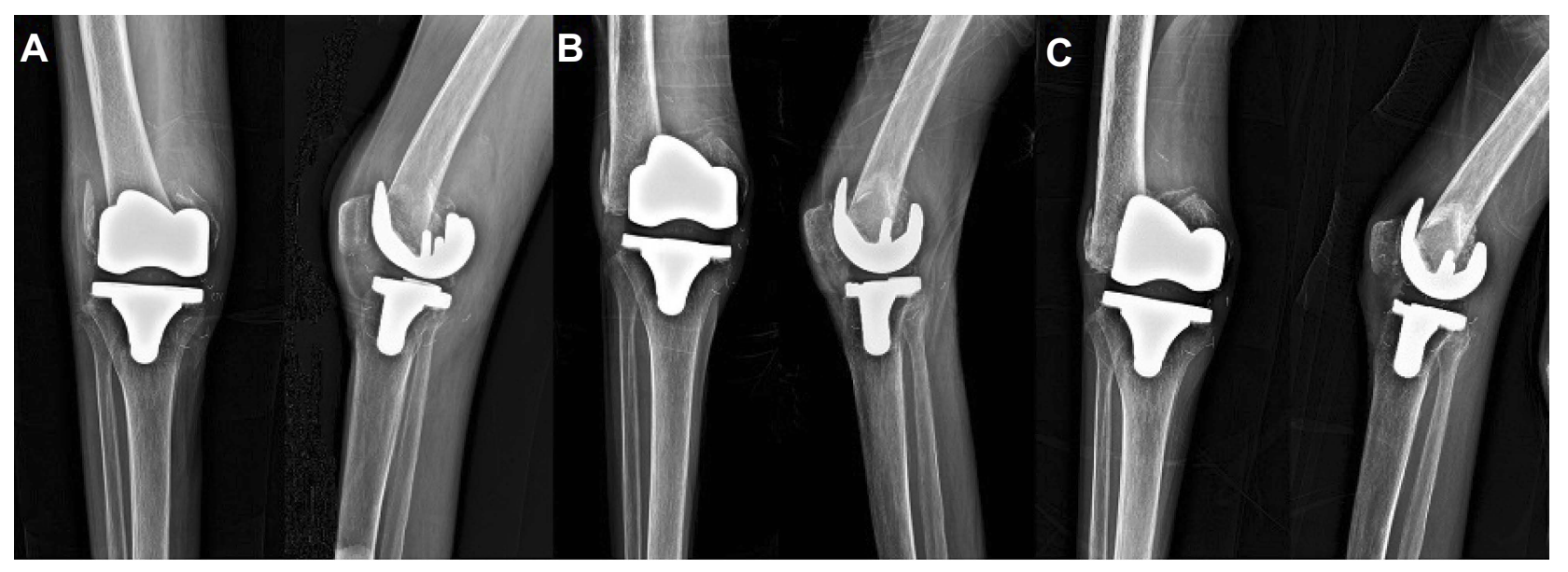

Figure 6 An 87-year-old woman was admitted to the hospital with a transfer fracture in the distal femur. (A) On simple radiography, a fracture with shortening to the upper part of the femur component in the total knee replacement state was observed. (B) A 10-week radiograph after the sponge cast was applied (C) A I6-week radiograph after sponge casting, which shows that union of the fracture was not prominent, but no pseudo-motion remained.

identified in 11 cases, which was confirmed at an average of 20.2 weeks (range: 16-28 weeks). There were four cases of non-complete bony union there was no callus formation around the fracture, but the patients did not complain of pain and and no passive or active pseudo-motion. Of the remaining cases, pseudo-motion remained clinically in two cases (Table 2).

\section{Discussion}

The common complications observed in bedridden patients are muscle weakness (atrophy) and bone demineralization (osteoporosis); it has been well documented that bone loss is accelerated in patients with long-term confinement to bed or immobility. ${ }^{4}$ Two important factors reducing the mass and the quality of bone because of immobility are chronic disease and malnutrition. ${ }^{5}$ The development of osteoporosis may be a cause of the increased incidence of bone fracture. ${ }^{6}$ In a study of osteoporosis in patients in a bedridden state with hemiparesis, Taketo et al reported an approximate $10 \%$ reduction in bone mineral density on the paretic side compared with the nonparetic side. In particular, joint contractures are often seen in bedridden patients when the immobilization period is long. . Joint contracture is another potential risk for fracture; ${ }^{7}$ the

Table 2 Clinical and radiological results

\begin{tabular}{|l|l|l|l|}
\hline Parameter & & Results & \\
\hline Clinical results & Under passive ROM & 15 cases & \\
\hline Pseudo-motion improvement & Timing (weeks) & $17.3(14-23)$ & \\
\hline & Under active ROM & 17 cases & Periprosthetic fx (knee): I case \\
\hline Pseudo-motion remaining & Under passive ROM & 2 cases & Femur neck fx: I case \\
\hline & & & \\
\hline Radiological results & & & \\
\hline Bony union & & 11 cases & \\
\hline & Timing (weeks) & $20.2(16-28)$ & cases \\
\hline Fibrous union & & 2 cases & \\
\hline Pseudo-motion remaining & & & \\
\hline
\end{tabular}

Abbreviations: ROM, range of motion; $f x$, fracture. 
external forces caused by changing the patient's position could cause fractures of the long bones.

These types of fractures occur in elderly patients, especially those with functional deficiency, in a nursing home. ${ }^{8}$ Although vertebral bone fractures or hip bone fractures were frequently reported in elderly care centers, ${ }^{9,10}$ few reports regarding long bone fractures in the elderly in bedridden states are available. Transfer fractures of the long bones (humerus, femur, tibia, fibula, and ulna) are frequently caused by direct force or the momentum force used for the displacement of the patient to prevent bedsores. In another study conducted by Martin-Hunyadi et al with 3,025 elderly patients for 20 months in elderly care centers, the rate of transfer fractures was approximately $1 \% ;{ }^{11}$ most were in the hip and in the shaft of the femur, tibia, fibula, and humerus. In another epidemiological study in elderly care centers, the rate of transfer fractures was reported to be $0.8 \%$ in 1 year, ${ }^{5}$ and the most common fractures were in the hip and in those with less movement in bedridden states. Regarding the risk factor for transfer fractures, Wong et al analyzed 30 cases and reported that female sex, bedridden state, nutrition tube, dementia, joint contracture, and hemiplegia of the same side were risk factors for fracture. ${ }^{12}$

When the transfer fractures occur in immobile patients, in order to reduce pain and allow position change and nursing care, a splint or cast is applied to reduce pseudomotion. However, bedridden patients are at a higher risk of cast-related complications. These include patients with an inability to effectively communicate to prevent increased areas of pressure on and constriction of the limb during cast immobilization. ${ }^{13,14}$ Because of the synthetic fiberglass materials and their increased stiffness, special precautions are recommended when applying a fiberglass cast to prevent increased areas of pressure on and constriction of the limb. ${ }^{15}$ However, despite caution, bedridden patients have a high incidence of pressure sores after cast immobilization. Instead of immobilization materials, such as synthetic fiberglass with high stiffness, a sponge cast that is porous, light-weighted, and exerts no pressure on the skin was used in the present study, and no occurrence of pressure sores was reported. In addition to the advantages of the material itself, it was considered that the change of position and maintenance of personal hygiene were easy, and the material itself was simple to replace. Therefore, the advantages of the sponge cast are that it does not cause pressure sores, it is light, and it has an excellent immobilizing ability; therefore, it can be summarized that it aids in the nursing care of patients, does not require special tools, and can be manufactured and replaced in a short period of time in a cost-effective way.

There were six cases (35.3\%) of artificial periarticular fracture in the study. The increasingly rapid transition to an older society, as marked by the increased number of elderly due to a prolonged average life span, is now a global phenomenon. There is a growing number of patients receiving artificial joint arthroplasty due to the aging society. ${ }^{16}$ In addition, metal implants are often inserted into the bone due to various reasons such as for hip fractures. ${ }^{17}$ As there have been no reports of previous fractures to the bone where the spontaneous fractured occurred, ${ }^{7}$ it is necessary for the care institution to accurately determine the patient's orthopedic history. Furthermore, the skeletal osteometry of Asian populations differs considerably from that of Western populations $;{ }^{18,19}$ bowing of the femur and tibia is common among Asians with end-stage osteoarthritis of the knee. For example, coronal bowing of the femur or tibia in Chinese patients with end-stage primary osteoarthritis of the knee is a common occurrence (femur, 62\%; tibia, 32\%) and can sometimes be severe (with the maximal lateral femoral bowing being $\left.20^{\circ}\right) .{ }^{20}$ There were also three cases of coronal bowing of the femur among the 15 patients with a femur fracture. Therefore, greater care should be taken when changing the position of a bedridden patient with bowing of the long bone of the lower limb.

This study has some limitations. First, the sample size was small and the design was retrospective. However, collecting data from several cases is difficult because conservative treatment for patients with transfer fracture is rare. Second, we were unable to compare our findings with the results of operative treatment in patients with transfer fracture.

\section{Conclusion}

In immobile patients with a combination of lower limb contractures and osteoporotic bones, a simple twisting force or mild contusion is sufficient to cause a transfer fracture. When transfer fractures develop, a sponge cast appears to be one of the effective treatment options available for bedridden or chair-bound patients due to its low risk of complications and satisfactory clinical results.

\section{Ethics approval and informed consent}

Informed consent was obtained verbally prior to the study. This was reviewed and approved by the Institutional Review Board (IRB) of Gwangju Veterans Hospital (IRB 
approval number: GJVH-IRB No. 2017-12-2) and the study was carried out in accordance with the latest version of the Declaration of Helsinki. Subjects of this study were first selected from ICD codes in the EMR system.

\section{Author contributions}

All authors contributed to data analysis, drafting or revising the article, gave final approval of the version to be published, and agree to be accountable for all aspects of the work.

\section{Disclosure}

The authors declare no conflicts of interest in this work.

\section{References}

1. Sherman FT. Transfer and turning fractures in nursing home patients. $A m J$ Med. 1991;91(6):668-669. doi:10.1016/0002-9343(91)90228-P

2. Kumar A, Kumar N. Prediction of fixation failure after sliding hip screw fixation. Injury. 2005;36(8):984-985. doi:10.1016/j.injury.2004.12.042

3. Richmond J, Aharonoff GB, Zuckerman JD, Koval KJ. Mortality risk after hip fracture. J Orthop Trauma. 2003;17(1):S2-S5. doi:10.1097/ 00005131-200309001-00002

4. Kane RS, Goodwin JS. Spontaneous fractures of the long bones in nursing home patients. Am J Med. 1991;90(2):263-266. doi:10.1016/ 0002-9343(91)90553-A

5. Kane RS, Burns EA, Goodwin JS. Minimal trauma fractures in older nursing home residents: the interaction of functional status, trauma, and site of fracture. $J$ Am Geriatr Soc. 1995;43(2):156-159. doi:10.1111/j.1532-5415.1995.tb06381.x

6. Takamoto S, Masuyama T, Nakajima M, et al. Alterations of bone mineral density of the femurs in hemiplegia. Calcif Tissue Int. 1995;56(4):259-262.

7. Takamoto S, Saeki S, Yabumoto Y, et al. Spontaneous fractures of long bones associated with joint contractures in bedridden elderly inpatients: clinical features and outcome. J Am Geriatr Soc. 2005;53 (8):1439-1441. doi:10.1111/j.1532-5415.2005.53433 3.x

8. Johnell O, Kanis JA. An estimate of the worldwide prevalence and disability associated with osteoporotic fractures. Osteoporos Int. 2006;17(12):1726-1733. doi:10.1007/s00198-006-0172-4
9. Rodondi A, Chevalley T, Rizzoli R. Prevalence of vertebral fracture in oldest old nursing home residents. Osteoporos Int. 2012;23 (11):2601-2606. doi:10.1007/s00198-012-1900-6

10. Benzinger P, Becker C, Kerse N, et al. Pelvic fracture rates in community-living people with and without disability and in residents of nursing homes. J Am Med Dir Assoc. 2013;14(9):673-678. doi:10.1016/j.jamda.2013.03.012

11. Martin-Hunyadi C, Heitz D, Kaltenbach G, et al. Spontaneous insufficiency fractures of long bones: a prospective epidemiological survey in nursing home subjects. Arch Gerontol Geriatr. 2000;31 (3):207-214. doi:10.1016/S0167-4943(00)00081-9

12. Wong TC, Wu WC, Cheng HS, Cheng YC, Yam SK. Spontaneous fractures in nursing home residents. Hong Kong Med J. 2007;13 (6):427-429.

13. Ragnarsson KT, Sell GH. Lower extremity fractures after spinal cord injury: A retrospective study. Arch Phys Med Rehabil. 1981;62 (9):418-423.

14. Sobel M, Lyden JP. Long bone fracture in a spinal cord injured patient: complication of treatment. A case report and review of the literature. $J$ Trauma. 1991;31(10):1440-1444. doi:10.1097/ 00005373-199110000-00026

15. Davids JR, Frick SL, Skewes E, Blackhurst DW. Skin surface pressure beneath an above the knee cast: plaster casts compared with fiberglass casts. J Bone Joint Surg Am. 1997;79(4):565-569. doi:10.2106/00004623-199704000-00013

16. Rayan F, Haddad F. Periprosthetic femoral fractures in total hip arthroplasty - a review. Hip Int. 2010;20(4):418-426. doi:10.1177/ 112070001002000402

17. Mann E, Icks A, Haastert B, Meyer G. Hip fracture incidence in the elderly in Austria: an epidemiological study covering the years 1994 to 2006. BMC Geriatr. 2008;8:35. doi:10.1186/14712318-8-35

18. Tang WM, Chiu KY, Kwan MF, Ng TP, Yau WP. Sagittal bowing of the distal femur in Chinese patients who require total knee arthroplasty. J Orthop Res. 2005;23(1):41-45. doi:10.1016/j. orthres.2004.06.013

19. Yip DK, Zhu YH, Chiu KY, Ng TP. Distal rotational alignment of the Chinese femur and its relevance in total knee arthroplasty. $J$ Arthroplasty. 2004;19(5):613-619.

20. Yau WP, Chiu KY, Tang WM, Ng TP. Coronal bowing of the femur and tibia in Chinese: its incidence and effects on total knee arthroplasty planning. J Orthop Surg (Hong Kong). 2007;15(1):32-36. doi: $10.1177 / 230949900701500108$
Clinical Interventions in Aging

\section{Publish your work in this journal}

Clinical Interventions in Aging is an international, peer-reviewed journal focusing on evidence-based reports on the value or lack thereof of treatments intended to prevent or delay the onset of maladaptive correlates of aging in human beings. This journal is indexed on PubMed Central, MedLine, CAS, Scopus and the Elsevier
Bibliographic databases. The manuscript management system is completely online and includes a very quick and fair peer-review system, which is all easy to use. Visit http://www.dovepress.com/ testimonials.php to read real quotes from published authors. 